\title{
Multi-modal Imaging of Plant-microbe Interactions in the Pine Rhizosphere
}

RYAN TAPPERO ${ }^{1 *}$, SUNNY LIAO ${ }^{2}$, JENNY BHATNAGAR ${ }^{3}$, COLIN AVERILL ${ }^{3}$, KOKO $\mathrm{CHEN}^{2}$, KAILE ZHANG ${ }^{2}$, SARAH L NICHOLAS $^{1}$, HUGH O’NEILL ${ }^{4}$, HASSINA BILHEUX ${ }^{4}$, RYTAS VILGALYS 5

${ }^{1}$ Brookhaven National Laboratory, Upton, NY 11973;

(*correspondence: rtappero@bnl.gov)

${ }^{2}$ University of Florida, Quincy, FL 32351;

${ }^{3}$ Boston University, Boston, MA 02215;

${ }^{4}$ Oak Ridge National Laboratory, Oak Ridge, TN 37831 ;

${ }^{5}$ Duke University, Durham, NC 27708
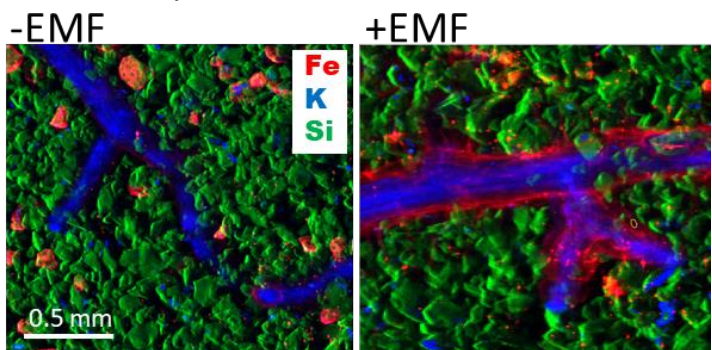

Transfers of iron (Fe) and other micronutrients (e.g., $\mathrm{Zn}, \mathrm{Cu}, \mathrm{Mn}$ ) from the soil to the plant root by ectomycorrhizal fungi (EMF) is a critical process in the rhizosphere that enhances primary productivity on marginal soils and influences production cost of biofeedstocks. Our experiment with Pinus-Suillus seeks explicit quantification of the (micro)nutrient dynamics across the plantmycorrhiza-soil interface with the goal of establishing the role of ectomycorrhizal symbiosis in iron acquisition. Microcosms were planted with sand culture supplemented with "point sources" of iron (ferrihydrite coated sand). Live-plant X-ray fluorescence imaging revealed differences between + EMF and -EMF treatments. Microcosms without host-specific EMF contained predominantly unaltered grains of iron-coated sand following 2 months growth while those with EMF contained severely altered grains that had largely 'disintegrated', and roots had fungal sheath enriched with iron. Spatially-resolved X-ray absorption spectroscopy identified an $\mathrm{Fe}$ (III) organometallic complex associated with the fungal sheath. Morphological changes to roots were visibly evident for EMF treatments. Attenuationbased neutron radiography and tomography are underway to characterize root architecture and foraging behavior $(+\mathrm{Fe} /-\mathrm{Fe})$ in marginal soil. 
This abstract is too long to be accepted for publication.

Please revise it so that it fits into the column on one page.

Alternate title:

Biogeochemical alteration of iron in the pine (mycor)rhizosphere 